\title{
Slide multiplicity free key polynomials
}

\author{
Soojin Cho* \\ Department of Mathematics \\ Ajou University \\ Suwon 16499, Korea \\ chosj@ajou.ac.kr
}

\author{
Stephanie van Willigenburg ${ }^{\dagger}$ \\ Department of Mathematics \\ University of British Columbia \\ Vancouver BC V6T 1Z2, Canada \\ steph@math.ubc.ca
}

Submitted: Sep 12, 2020; Accepted: Jan 7, 2022; Published: Jan 28, 2022

(C) The authors. Released under the CC BY-ND license (International 4.0).

\begin{abstract}
Schubert polynomials are refined by the key polynomials of Lascoux-Schützenberger, which in turn are refined by the fundamental slide polynomials of AssafSearles. In this paper we determine which fundamental slide polynomial refinements of key polynomials, indexed by strong compositions, are multiplicity free. We also give a recursive algorithm to determine all terms in the fundamental slide polynomial refinement of a key polynomial indexed by a strong composition. From here, we apply our results to begin to classify which fundamental slide polynomial refinements, indexed by weak compositions, are multiplicity free. We completely resolve the cases when the weak composition has at most two nonzero parts or the sum has at most two nonzero terms.
\end{abstract}

Mathematics Subject Classifications: 05E05, 05E10, 14N15

\section{Introduction}

Schubert polynomials were introduced by Lascoux-Schützenberger [13], and represent cohomology classes of Schubert cycles in flag varieties. They are also generalizations of the well-known Schur polynomials and are generating functions for pipe dreams. LascouxSchützenberger showed in [14] that Schubert polynomials expand as a positive linear combination of key polynomials, which are not only a tool for studying Schubert polynomials but also arise as characters for the Demazure modules of type $A[12,15,16]$. Recently multiplicity free key polynomials in terms of monomials have arisen in the study of spherical Schubert geometry [10] and multiplicity free key polynomials in terms of monomials were recently succinctly classified by Hodges-Yong in [11].

\footnotetext{
* Supported by the Ajou University research fund.

${ }^{\dagger}$ Supported by the National Sciences and Engineering Research Council of Canada.
} 
This result joins a myriad of other multiplicity free classifications in algebraic combinatorics including multiplicity free products of Schur functions in terms of Schur functions [18], and analogously for multiplicity free Schur $P$-function products [3], multiplicity free skew Schur functions in terms of Schur functions [9, 19], multiplicity free Schur $P$-functions in terms of Schur functions [17], multiplicity free Stanley symmetric functions in terms of monomials [5], multiplicity free Schubert polynomials in terms of monomials [7], and multiplicity free Schur functions in terms of fundamental quasisymmetric functions [4]. This latter result is as follows.

Theorem 1 (Theorem 3.3, [4]). For $\lambda$ a partition of $n$, the Schur function $s_{\lambda}$ has a multiplicity free expansion into a sum of fundamental quasisymmetric functions if and only if $\lambda$ or its transpose is one of

(1) $(3,3)$ if $n=6$,

(2) $(4,4)$ if $n=8$,

(3) $(n-2,2)$ if $n \geqslant 4$,

(4) $\left(n-k, 1^{k}\right)$ if $n \geqslant 1$ and $0 \leqslant k \leqslant n-1$.

Fundamental quasisymmetric functions themselves are an active area of study, being the weight enumerators of chains in the theory of $P$-partitions [8] and being isomorphic to the irreducible characters of the 0-Hecke algebra [6]. They are also the stable limits of fundamental slide polynomials [2, Theorem 4.4] that were introduced by Assaf-Searles to study Schubert polynomials [1] who similarly showed that key polynomials are the stable limits of Schur functions [2, Corollary 4.9].

Since Schur functions and fundamental quasisymmetric functions are stable limits of key polynomials and fundamental slide polynomials respectively, as described in Theorem 6 , it is natural to ask if we can classify key polynomials that are expanded as a multiplicity free sum of fundamental slide polynomials. In this paper we consider the multiplicity free expansion of key polynomials into fundamental slide polynomials, and obtain the following classification in Theorem 26.

Theorem. For $\alpha$ a strong composition, the key polynomial $\kappa_{\alpha}$ has a multiplicity free expansion into a sum of fundamental slide polynomials if and only if $\alpha=\left(\alpha_{1}, \ldots, \alpha_{\ell}\right)$ satisfies all three of the following conditions:

(a) There is no $i<j<k$ such that $\alpha_{i}<\alpha_{j}<\alpha_{k}$.

(b) There is no $i<j<k<l$ such that $\alpha_{i}, \alpha_{j}<\alpha_{l}<\alpha_{k}$.

(c) There is no $i<j<k<l$ such that $\alpha_{i}, \alpha_{j}+1<\alpha_{k}=\alpha_{l}$.

More precisely our paper is structured as follows. In Section 2 we recall the relevant background before classifying when a key polynomial is equal to a fundamental slide polynomial in Theorem 13 and the sum of two fundamental slide polynomials in Theorem 22 . 
We then restrict our attention to key polynomials indexed by strong compositions and their multiplicity free expansion into fundamental slide polynomials in Section 4, generalizing Theorem 1. In particular we classify this in Theorem 26 and give an algorithm to compute the terms in general in Subsection 4.1. In Section 5 we work towards a full classification, including when a key polynomial whose index has two nonzero parts is a multiplicity free expansion of fundamental slide polynomials in Subsection 5.1.

\section{Tableaux and polynomials}

In this section we recall the background needed in order to state and prove our results.

A weak composition $\mathbf{a}=\left(a_{1}, \ldots, a_{\ell}\right)$ of length $\ell$ is a finite sequence of nonnegative integers, and a strong composition $\alpha=\left(\alpha_{1}, \ldots, \alpha_{\ell}\right)$ of length $\ell$ is a finite sequence of positive integers. For a weak composition a, we let flat(a) be the strong composition obtained by removing zeros from a and $\operatorname{sort}(\mathbf{a})$ be the partition obtained by rearranging the parts of flat(a) into weakly decreasing order. For example, if $\mathbf{a}=(0,0,2,3)$ then flat $(\mathbf{a})=(2,3)$ and $\operatorname{sort}(\mathbf{a})=(3,2)$. Given two weak compositions $\mathbf{a}=\left(a_{1}, \ldots, a_{\ell}\right)$ and $\mathbf{b}=\left(b_{1}, \ldots, b_{\ell}\right)$ we say that $\mathbf{b}$ dominates $\mathbf{a}$ denoted by $\mathbf{b} \geqslant \mathbf{a}$, if $b_{1}+\cdots+b_{i} \geqslant a_{1}+\cdots+a_{i}$ for all $i=1, \ldots, \ell$. Given two strong compositions $\alpha=\left(\alpha_{1}, \ldots, \alpha_{\ell}\right)$ and $\beta=\left(\beta_{1}, \ldots, \beta_{\ell^{\prime}}\right)$, we say that $\beta$ refines $\alpha$ if there is a sequence $0=i_{0}<i_{1}<i_{2}<\cdots<i_{\ell}=\ell^{\prime}$ such that $\beta_{i_{j-1}+1}+\cdots+\beta_{i_{j}}=\alpha_{j}$ for $j=1, \ldots, \ell$.

A diagram is a finite subset of $\mathbb{N} \times \mathbb{N}$ where the coordinate $(i, j)$ represents the cell at the $i$ th row from the bottom and the $j$ th column from the left.

We now come to two central definitions, which define the tableaux that we will be using.

Definition 2. For a weak composition $\mathbf{a}=\left(a_{1}, \ldots, a_{\ell}\right)$, a Kohnert tableau of content $\mathbf{a}$ is a diagram filled with $a_{i}$ is for $i=1, \ldots, \ell$, satisfying the following conditions:

(i) For each $i=1, \ldots, \ell$, there is exactly one $i$ in each column from 1 through $a_{i}$.

(ii) Each entry in row $i$ is at least $i$ for all $i$.

(iii) For each $i=1, \ldots, \ell$, the cells filled with $i$ weakly descend from left to right.

(iv) If $i<j$ appear in a column with $i$ above $j$, then there is an $i$ in the column immediately to the right of and strictly above the cell containing the $j$.

A Kohnert tableau of content $\mathbf{a}$ is called a quasi-Yamanouchi Kohnert tableau if it satisfies an additional condition:

(v) If a row is not empty, say the $i$ th row, then

- either there is a cell filled with $i$ in the $i$ th row,

- or there is a cell in the $(i+1)$ th row that lies weakly right of a cell in the $i$ th row. 
Definition 3. For a weak composition $\mathbf{a}=\left(a_{1}, \ldots, a_{\ell}\right)$, let $\mathrm{KT}(\mathbf{a})$ be the set of Kohnert tableaux and $\operatorname{QKT}(\mathbf{a})$ be the set of quasi-Yamanouchi Kohnert tableaux of content a. For $T \in \mathrm{KT}(\mathbf{a})$ we let $\operatorname{wt}(T)$ be the weak composition whose $i$ th part is the number of cells in the $i$ th row of $T$. The basic quasi-Yamanouchi Kohnert tableau of content $\mathbf{a}$ is the unique Kohnert tableau $T$ with $a_{i}$ cells in row $i$ filled with $i$ for $i=1, \ldots, \ell$, namely $\operatorname{wt}(T)=\mathbf{a}$.

Example 4. Figure 1 illustrates some Kohnert tableaux that are quasi-Yamanouchi. Note that the leftmost one is basic. Figure 2 illustrates some Kohnert tableaux that are not quasi-Yamanouchi.
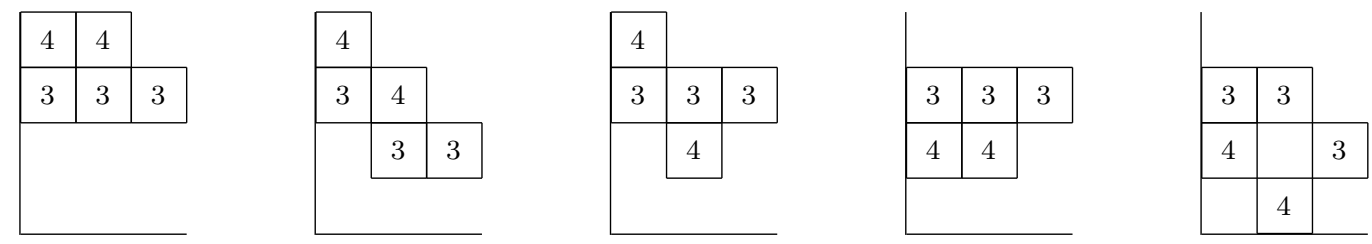

Figure 1: Quasi-Yamanouchi Kohnert tableaux of content $(0,0,3,2)$.
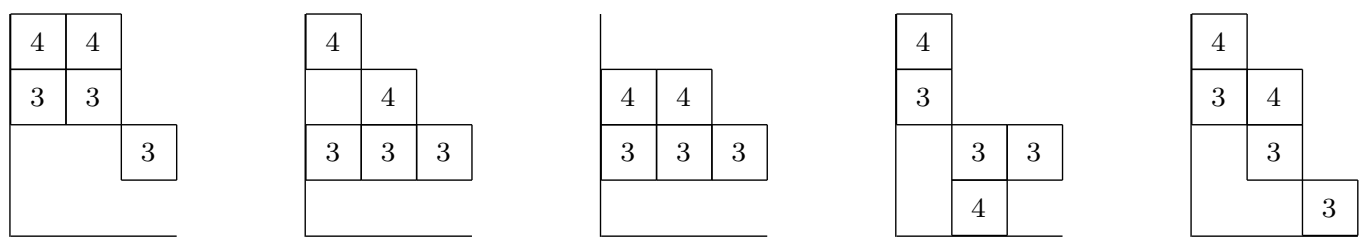

Figure 2: Some Kohnert tableaux of content $(0,0,3,2)$ that are not quasi-Yamanouchi.

For a weak composition $\mathbf{b}=\left(b_{1}, \ldots, b_{\ell}\right)$, we use $x^{\mathbf{b}}$ to denote the monomial $x_{1}^{b_{1}} \cdots x_{\ell}^{b_{\ell}}$. We are now ready to define key polynomials, fundamental slide polynomials, each of their stable limits, and the relationship between them.

Definition 5. For a weak composition a of length $\ell$,

(1) the key polynomial $\kappa_{\mathbf{a}}=\kappa_{\mathbf{a}}\left(x_{1}, \ldots, x_{\ell}\right)$ is defined as

$$
\kappa_{\mathbf{a}}=\sum_{T \in \mathrm{KT}(\mathbf{a})} x^{\mathrm{wt}(T)},
$$

(2) the fundamental slide polynomial $\mathfrak{F}_{\mathbf{a}}=\mathfrak{F}_{\mathbf{a}}\left(x_{1}, \ldots, x_{\ell}\right)$ is defined as

$$
\mathfrak{F}_{\mathbf{a}}=\sum_{\substack{\mathbf{b} \geqslant \mathbf{a} \\ \text { flat }(\mathbf{b}) \text { refines flat }(\mathbf{a})}} x^{\mathbf{b}} .
$$


If we let $s_{\lambda}$ denote the Schur function indexed by the partition $\lambda$ and $F_{\alpha}$ denote the fundamental quasisymmetric function indexed by the strong compsition $\alpha$, then we have the following.

Theorem 6 ([2]). For a weak composition $\mathbf{a}$, we have

$$
\begin{aligned}
& \lim _{m \rightarrow \infty} \kappa_{0^{m} \times \mathbf{a}}=s_{\text {sort }(\mathbf{a})}, \\
& \lim _{m \rightarrow \infty} \mathfrak{F}_{0^{m} \times \mathbf{a}}=F_{\text {flat }(\mathbf{a})},
\end{aligned}
$$

where $0^{m} \times \mathbf{a}$ means prepending $m 0 s$ to $\mathbf{a}$.

Proposition 7 (Theorem 2.13, [2]). For a weak composition a, the key polynomial indexed by $\mathbf{a}$ is

$$
\kappa_{\mathbf{a}}=\sum_{T \in \mathrm{QKT}(\mathbf{a})} \mathfrak{F}_{\mathrm{wt}(T)}
$$

Example 8. In Figure 1, all quasi-Yamanouchi Kohnert tableaux of content $(0,0,3,2)$ are given, and due to Proposition 7 we have

$$
\kappa_{(0,0,3,2)}=\mathfrak{F}_{(0,0,3,2)}+\mathfrak{F}_{(0,2,2,1)}+\mathfrak{F}_{(0,1,3,1)}+\mathfrak{F}_{(0,2,3,0)}+\mathfrak{F}_{(1,2,2,0)} .
$$

\section{$3 \quad$ Initial results}

We now prove some initial multiplicity free classifications, which will be useful later. In particular we classify when a key polynomial is equal to a fundamental slide polynomial or the sum of two fundamental slide polynomials. In each case we give the precise decomposition.

\subsection{Classifying when $\kappa_{\mathrm{a}}=\mathfrak{F}_{\mathrm{a}}$}

Before establishing our classification we prove four lemmas.

Lemma 9. Let $\mathbf{a}=(a_{1}, \ldots, a_{k}, \underbrace{0, \ldots, 0}_{m}, a_{k+m+1})$ for positive integers $a_{1} \geqslant \cdots \geqslant a_{k}, a$ nonnegative integer $a_{k+m+1} \leqslant a_{k}$ and a nonnegative integer $m$. Then $\kappa_{\mathbf{a}}=\mathfrak{F}_{\mathbf{a}}$.

Proof. The basic quasi-Yamanouchi Kohnert tableau of content $\mathbf{a}$ is the unique element of $\operatorname{QKT}(\mathbf{a})$. Due to Proposition 7, we can conclude that $\kappa_{\mathbf{a}}=\mathfrak{F}_{\mathbf{a}}$.

Lemma 10. Let $\mathbf{a}=\left(a_{1}, \ldots, a_{k}, \bar{a}_{01}, 1\right)$ for positive integers $a_{1} \geqslant \cdots \geqslant a_{k}>1$ and $\bar{a}_{01} a$ finite sequence of $0 s$ and 1 s. Then $\kappa_{\mathbf{a}}=\mathfrak{F}_{\mathbf{a}}$.

Proof. The basic quasi-Yamanouchi Kohnert tableau of content $\mathbf{a}$ is the unique element of $\operatorname{QKT}(\mathbf{a})$. Due to Proposition 7, we can conclude that $\kappa_{\mathbf{a}}=\mathfrak{F}_{\mathbf{a}}$. 
Lemma 11. If a weak composition $\mathbf{a}=\left(a_{1}, \ldots, a_{\ell}\right)$ has two nonzero entries $0<a_{i}<a_{j}$ with $i<j$, then the key polynomial $\kappa_{\mathbf{a}}$ has at least two different terms in its expansion into fundamental slide polynomials.

Proof. Choose $i<j$ with $0<a_{i}<a_{j}$ so that $(j-i)$ is minimal. Then $a_{i+1}=\cdots=a_{j-1}=$ 0 , and moving the last cell of row $j$ of the basic quasi-Yamanouchi Kohnert tableau down to row $i$ produces another tableau in $\operatorname{QKT}(\mathbf{a})$.

Lemma 12. Suppose that a weak composition $\mathbf{a}=\left(a_{1}, \ldots, a_{\ell}\right)$ has two nonzero entries $a_{i}, a_{j}$ with $i<j$ and at least one of $a_{i}$ and $a_{j}$ is strictly bigger than 1 . If there is $h<i$ such that $a_{h}=0$, then the key polynomial $\kappa_{\mathbf{a}}$ has at least two different terms in its expansion into fundamental slide polynomials. Moreover, if $a_{i} \neq 1$ then one term $\mathfrak{F}_{\left(b_{1}, \ldots, b_{\ell}\right)}$ has $b_{h} \neq 0$.

Proof. Let $h$ be the smallest index such that $a_{h}=0$ but $a_{h+1} \neq 0$, and let $i=h+1$. Then, by assumption we can find the smallest $j>h+1$ with $a_{j}>0$ so that at least one of $a_{i}, a_{j}$ is strictly bigger than 1 . There are three cases to consider.

If $1=a_{i}<a_{j}$, then the result follows by Lemma 11 .

If $1<a_{i} \leqslant a_{j}$, then $a_{k}=0$ for all $k=i+1, \ldots, j-1$. Therefore, moving the last $i$ in row $i$ down to row $i-1=h$ and the last $j-i+1$ entries in row $j$ down to row $i$ from the basic quasi-Yamanouchi Kohnert tableau will produce another tableau in QKT(a). See Figure 3.

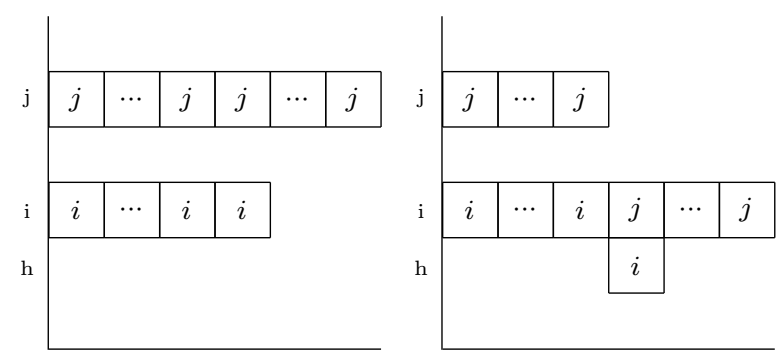

Figure 3: Two quasi-Yamanouchi Kohnert tableaux when $1<a_{i} \leqslant a_{j}$ and $a_{h}=0$.

If $a_{i}>a_{j} \geqslant 1$, then $a_{k}=0$ for all $k=i+1, \ldots, j-1$. Therefore, moving the last $j$ in row $j$ down to row $i-1=h$ from the basic quasi-Yamanouch Kohnert tableau will produce another tableau in $\mathrm{QKT}(\mathbf{a})$. See Figure 4.

Finally note that in both the $a_{i} \neq 1$ cases row $h$ has a cell in it.

Theorem 13. For a weak composition $\mathbf{a}$, the key polynomial $\kappa_{\mathbf{a}}$ has a unique term in the expansion into fundamental slide polynomials, that is $\kappa_{\mathbf{a}}=\mathfrak{F}_{\mathbf{a}}$, if and only if $\mathbf{a}$ is one of the following weak compositions.

(1) Every nonzero part of a is 1.

(2) a has only one nonzero part. 

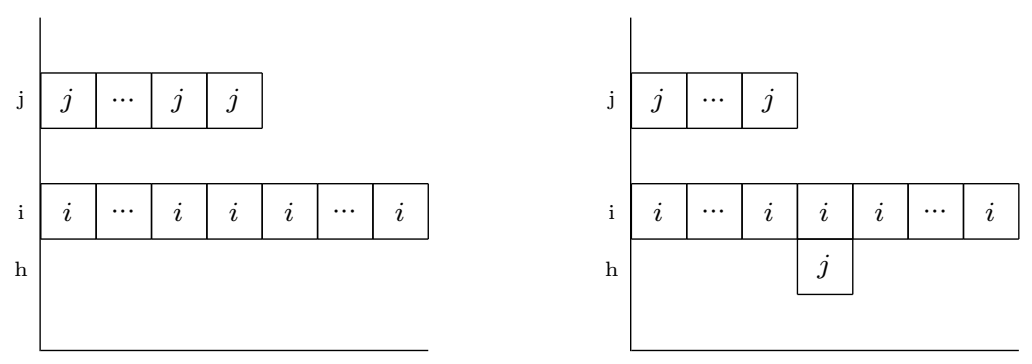

Figure 4: Two quasi-Yamanouchi Kohnert tableaux when $a_{i}>a_{j} \geqslant 1$ and $a_{h}=0$.

(3) $\mathbf{a}=(a_{1}, \ldots, a_{k}, \underbrace{0, \ldots, 0}_{m}, a_{k+m+1})$ for positive integers $a_{1} \geqslant \cdots \geqslant a_{k}$, a nonnegative integer $a_{k+m+1} \leqslant a_{k}$ and a nonnegative integer $m$.

(4) $\mathbf{a}=\left(a_{1}, \ldots, a_{k}, \bar{a}_{01}, 1\right)$ for positive integers $a_{1} \geqslant \cdots \geqslant a_{k}>1$ and $\bar{a}_{01}$ a finite sequence of $0 s$ and $1 s$.

Proof. It is easy to check that if either (1) or (2) is the case, then $\kappa_{\mathbf{a}}=\mathfrak{F}_{\mathbf{a}}$, and Lemma 9, Lemma 10, Lemma 11 and Lemma 12 complete the proof.

Example 14. If $\mathbf{a}=(3,0,0,2)$, then $\kappa_{(3,0,0,2)}=\mathfrak{F}_{(3,0,0,2)}$.

Corollary 15. For a strong composition $\alpha, \kappa_{\alpha}=\mathfrak{F}_{\alpha}$ if and only if $\alpha$ is a partition.

\section{$3.2 \quad$ Classifying when $\kappa_{\mathrm{a}}=\mathfrak{F}_{\mathrm{a}}+\mathfrak{F}_{\mathrm{b}}$}

We will first deal with the case of a strong composition and then apply this case to obtain the full result. We begin with two lemmas.

Lemma 16. For a strong composition $\alpha, \mathfrak{F}_{\alpha}$ and $\mathfrak{F}_{\operatorname{sort}(\alpha)}$ are always (not necessarily distinct) terms in $\kappa_{\alpha}$.

Proof. $\mathfrak{F}_{\alpha}$ comes from the basic quasi-Yamanouchi Kohnert tableau. $\mathfrak{F}_{\operatorname{sort}(\alpha)}$ comes from the quasi-Yamanouchi Kohnert tableau obtained from the basic one by bottom justifying each column with its entries written in increasing order from bottom to top. If $\alpha=\operatorname{sort}(\alpha)$, then these two quasi-Yamanouchi Kohnert tableaux are the same tableau.

Definition 17. For a strong composition $\alpha=\left(\alpha_{1}, \ldots, \alpha_{\ell}\right)$, a pair $(i, j), i<j$, is an inversion of $\alpha$ if $0<\alpha_{i}<\alpha_{j}$, and we let $\operatorname{inv}(\alpha)$ be the number of inversions of $\alpha$.

Example 18. If $\alpha=(2,3)$, then $\operatorname{inv}(\alpha)=1$.

Lemma 19. If $\operatorname{inv}(\alpha)=1$, then the unique inversion must be the pair $(i, i+1)$ for some $i$. If $\alpha=\left(\alpha_{1}, \ldots, \alpha_{i-1}, \alpha_{i}, \alpha_{i}+m, \alpha_{i+2}, \ldots, \alpha_{\ell}\right)$ where $m>0$ and $\alpha_{1} \geqslant \cdots \geqslant \alpha_{i} \geqslant \alpha_{i+2} \geqslant$ $\cdots \geqslant \alpha_{\ell}$, is a strong composition with only one inversion, then

$$
\kappa_{\alpha}=\sum_{t=0}^{m} \mathfrak{F}_{\left(\alpha_{1}, \ldots, \alpha_{i-1}, \alpha_{i}+t, \alpha_{i}+m-t, \alpha_{i+2}, \ldots, \alpha_{\ell}\right)} .
$$


Proof. If $\alpha_{i} \geqslant \alpha_{i+1}$ for all $i$, then $\operatorname{inv}(\alpha)=0$. Hence, if $\operatorname{inv}(\alpha)=1$ then $\alpha_{i}<\alpha_{i+1}$ must hold for some $i$ and this must be the unique such $i$, plus $\alpha_{i+1} \leqslant \alpha_{k}$ for all $1 \leqslant k \leqslant i-1$.

All Kohnert tableaux are the ones obtained by moving cells in row $(i+1)$ down to row $i$ one by one from the rightmost cell and these are all quasi-Yamanouchi, which proves the given equation.

We remark that the weight of $T \in \mathrm{QKT}(\alpha)$ for a strong composition $\alpha$, is a strong composition since row $i$ of the first column of any Kohnert tableau of content $\alpha$ is filled with $i$. Hence we know that if $\mathfrak{F}_{\mathbf{a}}$ appears in the expansion of $\kappa_{\alpha}$, then a must be a strong composition of the same length as $\alpha$.

Proposition 20. For a strong composition $\alpha, \kappa_{\alpha}=\mathfrak{F}_{\alpha}+\mathfrak{F}_{\beta}$ if and only if $\operatorname{inv}(\alpha)=1$ and $\alpha_{i+1}=\alpha_{i}+1$ for the unique inversion $(i, i+1)$ of $\alpha$. Furthermore, $\beta=\operatorname{sort}(\alpha)$ in this case.

Proof. Suppose that $\kappa_{\alpha}=\mathfrak{F}_{\alpha}+\mathfrak{F}_{\beta}$ for some strong composition $\beta$. Then, by Lemma 16, $\beta$ must be $\operatorname{sort}(\alpha)$ and $\operatorname{inv}(\alpha)>0$. Let $i$ be the smallest index such that $(i, i+1)$ is an inversion of $\alpha$. Then $\alpha_{i+1}=\alpha_{i}+1$ must hold, since otherwise at least three terms will appear in the expansion of $\kappa_{\alpha}$ into fundamental slide polynomials by moving cells in row $i+1$ down to row $i$ one by one from the rightmost cell. Now, if $\alpha_{i+2}>\alpha_{i}$ then either $\alpha_{i+2}>\alpha_{i+1}$ or $\alpha_{i+2}=\alpha_{i+1}$ must hold and in either case, by moving cells in row $i+1$ down to row $i$ and then moving cells in row $i+2$ down to row $i+1$, there are at least three terms in the expansion of $\kappa_{\alpha}$. Hence we have $\alpha_{i+2} \leqslant \alpha_{i}$. Since if there is $j \geqslant i+2$ such that $(j, j+1)$ is an inversion of $\alpha$, then this will make at least two more terms in the expansion of $\kappa_{\alpha}$, we can conclude that the only inversion of $\alpha$ is $(i, i+1)$ and $\alpha_{i+1}=\alpha_{i}+1$.

The proof of the other direction is immediate from Lemma 19.

Example 21. If $\alpha=(2,3)$, then $\kappa_{(2,3)}=\mathfrak{F}_{(2,3)}+\mathfrak{F}_{(3,2)}$.

We now apply our classification for strong compositions to obtain our full classification. We also need the following generalization of $\operatorname{sort}(\mathbf{a})$. For a weak composition a we let $\operatorname{sort}_{0}(\mathbf{a})$ be the weak composition whose nonzero parts are the parts of sort(a) taken in weakly decreasing order, and the $i$ th part of $\operatorname{sort}_{0}(\mathbf{a})$ is 0 if and only if the $i$ th part of $\mathbf{a}$ is 0 . For example, if $\mathbf{a}=(0,2,0,3)$ then $\operatorname{sort}_{0}(\mathbf{a})=(0,3,0,2)$.

Theorem 22. For a weak composition $\mathbf{a}, \kappa_{\mathbf{a}}=\mathfrak{F}_{\mathbf{a}}+\mathfrak{F}_{\mathbf{b}}$ if and only if $\mathbf{a}$ satisfies both the following conditions:

(a) $\operatorname{inv}(f l a t(\mathbf{a}))=1$ and flat $(\mathbf{a})_{i+1}=\operatorname{flat}(\mathbf{a})_{i}+1$ for the unique inversion $(i, i+1)$ of flat $(\mathbf{a})$,

(b) either

- $\operatorname{flat}(\mathbf{a})=(1,2)$, or

- $\mathbf{a}=(a_{1}, \ldots, a_{k}, \underbrace{0, \ldots, 0}_{m}, a_{k+m+1})$ for positive integers $a_{1}, \ldots, a_{k}$, a nonnegative integer $a_{k+m+1}$ and a nonnegative integer $m$, or 
- $\mathbf{a}=\left(a_{1}, \ldots, a_{k}, \bar{a}_{01}, 1\right)$ for positive integers $a_{1}, \ldots, a_{k}>1$ and $\bar{a}_{01}$ a finite sequence of $0 \mathrm{~s}$ and $1 \mathrm{~s}$.

Furthermore, $\mathbf{b}=\operatorname{sort}_{0}(\mathbf{a})$ in this case.

Proof. Let $\alpha$ be a strong composition with $\kappa_{\alpha} \neq \mathfrak{F}_{\alpha}+\mathfrak{F}_{\beta}$ and a be a weak composition such that $\operatorname{flat}(\mathbf{a})=\alpha$. Then $\kappa_{\mathbf{a}} \neq \mathfrak{F}_{\mathbf{a}}+\mathfrak{F}_{\mathbf{b}}$. This is because if $|\operatorname{QKT}(\alpha)|>2$ then by adding empty rows and increasing the numbers in the cells by the number of empty rows inserted below we will produce more than two tableaux in $\mathrm{QKT}(\mathbf{a})$.

Hence flat(a) must satisfy the conditions of Proposition 20, giving us the first condition. By Lemma 16 and noting that we can produce members in QKT(a) from $\operatorname{QKT}(\alpha)$ by adding empty rows and increasing the numbers in the cells by the number of empty rows inserted below, we know that $\kappa_{\mathbf{a}}=\mathfrak{F}_{\mathbf{a}}+\mathfrak{F}_{\text {sort }_{0}(\mathbf{a})}$ plus perhaps other terms. To complete the proof note that Lemma 12 then guarantees that we will have other terms unless flat $(\mathbf{a})=(1,2)$, or $\mathbf{a}=(a_{1}, \ldots, a_{k}, \underbrace{0, \ldots, 0}_{m}, a_{k+m+1})$ for positive integers $a_{1}, \ldots, a_{k}$, a nonnegative integer $a_{k+m+1}$ and a nonnegative integer $m$, or $\mathbf{a}=\left(a_{1}, \ldots, a_{k}, \bar{a}_{01}, 1\right)$ for positive integers $a_{1}, \ldots, a_{k}>1$ and $\bar{a}_{01}$ a finite sequence of $0 \mathrm{~s}$ and $1 \mathrm{~s}$. In this case, by the definition of quasi-Yamanouchi Kohnert tableaux and Proposition 7 no further terms are produced, giving us the second condition.

Example 23. If $\alpha=(2,0,0,3)$, then $\kappa_{(2,0,0,3)}=\mathfrak{F}_{(2,0,0,3)}+\mathfrak{F}_{(3,0,0,2)}$.

\section{Classifying when $\kappa_{\alpha}$ is multiplicity free for $\alpha$ a strong com- position}

In this section, we restrict our attention to strong compositions.

Theorem 24. Let $\alpha=\left(\alpha_{1}, \ldots, \alpha_{\ell}\right)$ be a strong composition.

(1) If there exist $i<j<k$ such that $\alpha_{i}<\alpha_{j}<\alpha_{k}$, then $\kappa_{\alpha}$ is not multiplicity free.

(2) If there exist $i<j<k<l$ such that $\alpha_{i}, \alpha_{j}<\alpha_{l}<\alpha_{k}$, then $\kappa_{\alpha}$ is not multiplicity free.

(3) If there exist $i<j<k<l$ such that $\alpha_{i}, \alpha_{j}+1<\alpha_{k}=\alpha_{l}$, then $\kappa_{\alpha}$ is not multiplicity free.

Example 25. The key polynomials $\kappa_{(1,2,3)}, \kappa_{(1,1,3,2)}$ and $\kappa_{(1,1,3,3)}$ are not multiplicity free.

Proof. Suppose that there are $i<j<k$ such that $\alpha_{i}<\alpha_{j}<\alpha_{k}$. We may assume that $k>j$ is the smallest $k$ such that $\alpha_{j}<\alpha_{k}$ and $i<j$ is the largest $i$ such that $\alpha_{i}<\alpha_{j}$, which imply that $\alpha_{x} \leqslant \alpha_{j}$ for all $j<x<k$ and $\alpha_{y} \geqslant \alpha_{j}$ for all $i<y<j$. The basic tableau of weight $\alpha$ is the first one in Figure 5 , in which $A$ is a subtableau contained in the first $\alpha_{j}$ columns. The second and the third tableaux of Figure 5 are quasi-Yamanouchi Kohnert tableaux of the same weight with content $\alpha$ and we can conclude that $\kappa_{\alpha}$ is not 
multiplicity free. We remark that the row filled with $*$ s in the first tableau of Figure 5 may not exist in which case the bottom two rows in the second and the third tableaux will be a single row without $*$ s.
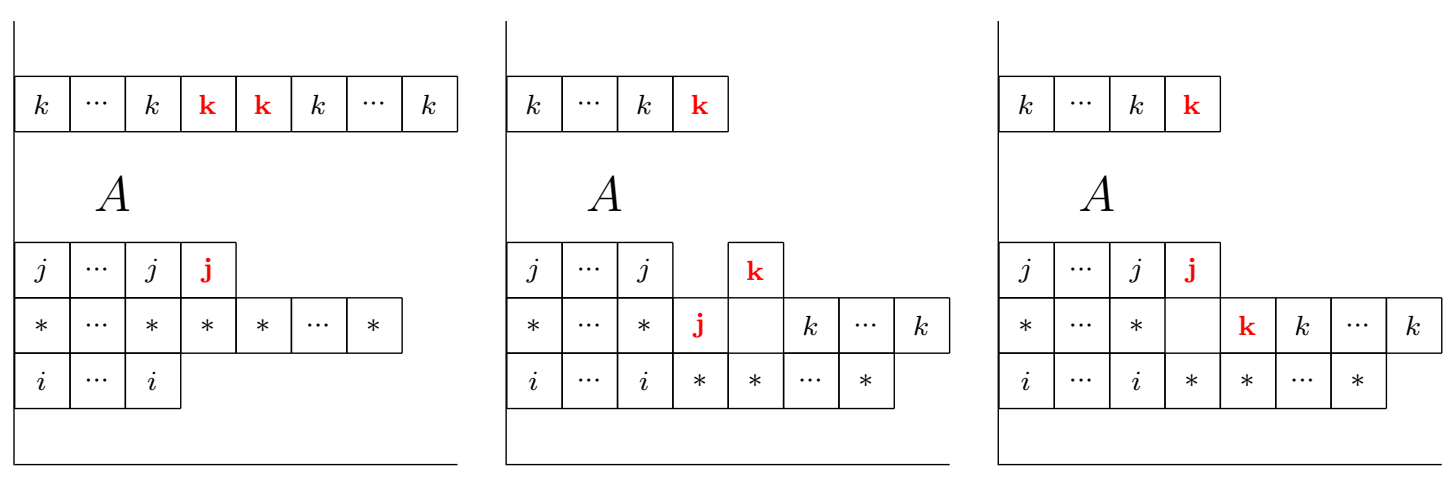

Figure 5: Basic tableau and two tableaux of same weight in $\operatorname{QKT}(\alpha)$ when $\alpha$ satisfies (1).

Now we will make a remark, which will be used often throughout the remainder of the proof, and will be referred to as "the remark above." We remark that if there is a row that is strictly longer than the rows we consider in a Kohnert tableau, then we can move cells across that row without violating any condition for being a quasi-Yamanouchi Kohnert tableau. We thus do not take account of the rows that are strictly longer than the rows we are considering.

Suppose that there are $i<j<k<l$ such that $\alpha_{i}, \alpha_{j}<\alpha_{l}<\alpha_{k}$. If there is $x>k$ such that $\alpha_{x}>\alpha_{k}$, then we have $\alpha_{j}<\alpha_{k}<\alpha_{x}$ and Case (1) is satisfied with $j<k<x$ and hence $\kappa_{\alpha}$ is not multiplicity free. Hence, we may assume that $\alpha_{x} \leqslant \alpha_{k}$ for all $x>k$, and $l>k$ is the smallest $l$ such that $\alpha_{l}<\alpha_{k}$. Then, $\alpha_{x}=\alpha_{k}$ for all $k<x<l$. On the other hand, if $\alpha_{i}<\alpha_{y}<\alpha_{k}$ for any $i<y<k$, then Case (1) is satisfied and hence $\kappa_{\alpha}$ is not multiplicity free. Therefore, for all $i<y<k, \alpha_{y} \leqslant \alpha_{i}$ so $\alpha_{j} \leqslant \alpha_{i}$ and moreover we can assume that $j=k-1$ and $i=k-2$. The basic tableau of weight $\alpha$ is the first one in Figure 6 for $\alpha_{i}=\alpha_{j}=\alpha_{\ell}-1$. The second and the third tableaux of Figure 6 are quasi-Yamanouchi Kohnert tableaux of the same weight with content $\alpha$ and we can conclude that $\kappa_{\alpha}$ is not multiplicity free. The figures for $\alpha_{j}<\alpha_{i}$, and other suitable $\alpha_{\ell}$, are almost identical.

Suppose that there are $i<j<k<l$ such that $\alpha_{i}, \alpha_{j}+1<\alpha_{k}=\alpha_{l}$. We choose the smallest $k$ and largest $j$ so that there is no $y$ such that $j<y<k$ and $\alpha_{y}=\alpha_{k}$ or $\alpha_{y}=\alpha_{j}$. We may assume there is also no $y$ such that $j<y<k$ and $\alpha_{j}<\alpha_{y}<\alpha_{k}$ since this is Case (1). Thus by the remark above we may assume that $\alpha_{y}<\alpha_{j}$ for all $j<y<k$. In Figure 7 we will not draw these rows, which we will fix.

Now we choose the smallest $l$ so that there is no $x$ such that $k<x<l$ and $\alpha_{x}=\alpha_{l}$. Then by the remark above we may assume that for all $k<x<l, \alpha_{x}<\alpha_{l}$. Furthermore we may assume $\alpha_{x} \leqslant \alpha_{j}$ for all $k<x<l$ to ensure there is no $x$ such that $j<x<l$ and 

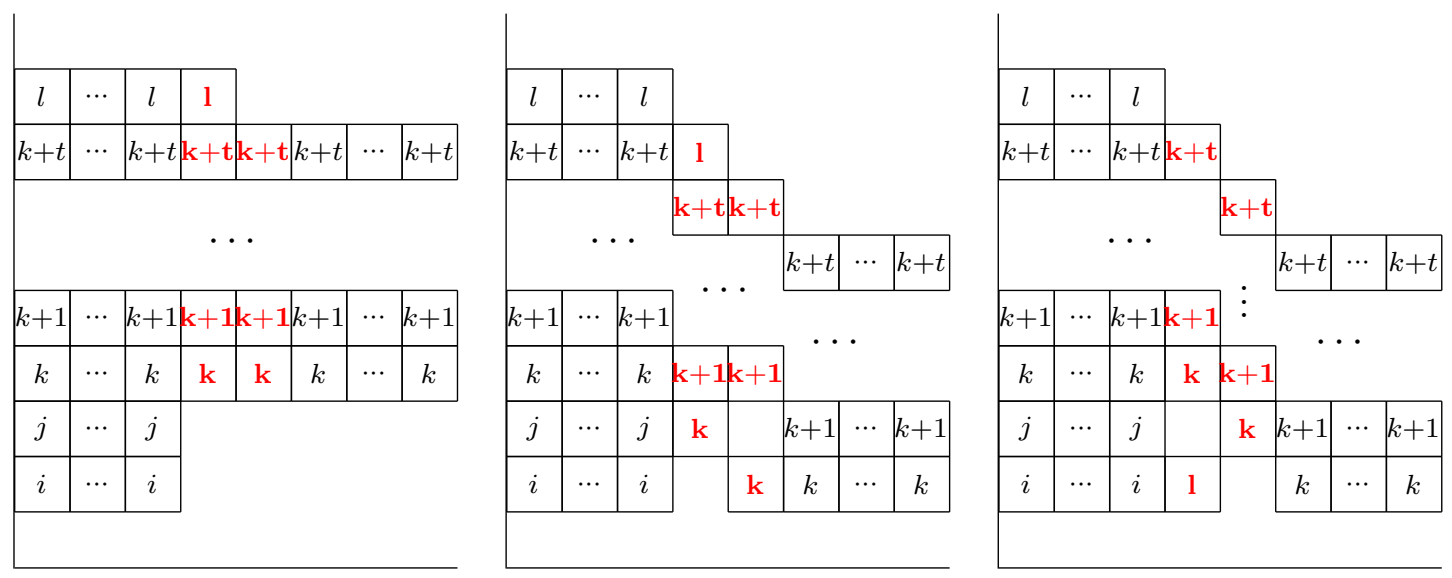

Figure 6: Basic tableau and two tableaux of same weight in $\operatorname{QKT}(\alpha)$ when $\alpha$ satisfies (2).

$\alpha_{j}<\alpha_{x}<\alpha_{l}$, since this is Case (1). Hence

$$
\alpha_{x} \leqslant \alpha_{j}<\alpha_{k}-1 \Rightarrow \alpha_{x} \leqslant \alpha_{k}-2
$$

In Figure 7 we will not draw these rows, which we will fix. Now we choose the largest $i$ so there is no $z$ such that $i<z<j$ and $\alpha_{z}<\alpha_{k}$. Furthermore we can assume that $\alpha_{z} \geqslant \alpha_{k}$ for all $i<z<j$ as otherwise we can choose $z<j<k<l$ such that $\alpha_{z}, \alpha_{j}+1<\alpha_{k}=\alpha_{l}$. Thus by the remark above we can assume that $\alpha_{z}=\alpha_{k}$. In Figure 7, we will denote the rows $i<z<j$ where $\alpha_{z}=\alpha_{k}$ collectively by $*$ and $\star$. We note that our construction also works if $j=i+1$.

We now consider the various cases for $\alpha_{i}$ and $\alpha_{j}$. We do not need to consider the case $\alpha_{i}<\alpha_{j}$ since this is Case (1). For the case $\alpha_{i}=\alpha_{j}+1$ we have that the basic tableau of weight $\alpha$ is the first one in Figure 7. The second and third tableaux of Figure 7 are quasi-Yamanouchi Kohnert tableaux of the same weight with content $\alpha$ and we can conclude in this case that $\kappa_{\alpha}$ is not multiplicity free. The figures for the cases $\alpha_{i}=\alpha_{j}$ and $\alpha_{i}>\alpha_{j}+1$ are almost identical.

We are now ready to give our classification of when $\kappa_{\alpha}$, for $\alpha$ a strong composition, is multiplicity free, and see that the conditions in Theorem 24 classify when $\kappa_{\alpha}$ has multiplicities.

Theorem 26. $\kappa_{\alpha}$ is multiplicity free if and only if $\alpha$ satisfies all three of the following conditions:

(a) There is no $i<j<k$ such that $\alpha_{i}<\alpha_{j}<\alpha_{k}$.

(b) There is no $i<j<k<l$ such that $\alpha_{i}, \alpha_{j}<\alpha_{l}<\alpha_{k}$.

(c) There is no $i<j<k<l$ such that $\alpha_{i}, \alpha_{j}+1<\alpha_{k}=\alpha_{l}$. 

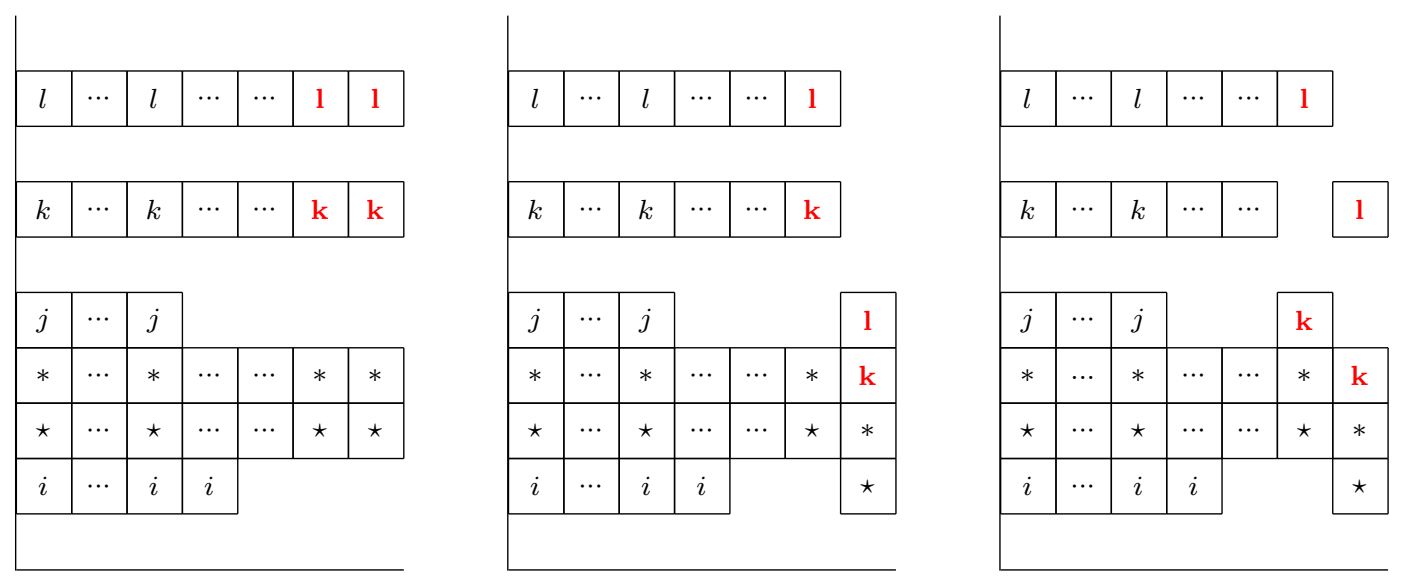

Figure 7: Basic tableau and two tableaux of same weight in $\operatorname{QKT}(\alpha)$ when $\alpha$ satisfies (3).

Proof. We use an induction on the number $\ell(\alpha)$ of parts of $\alpha$.

If $\ell(\alpha)=1$ or $\ell(\alpha)=2$ and $\operatorname{inv}(\alpha)=0$, then $\kappa_{\alpha}=\mathfrak{F}_{\alpha}$ by Corollary 15. If $\ell(\alpha)=2$ and $\operatorname{inv}(\alpha)=1$ then by Lemma $19 \kappa_{\alpha}$ is multiplicity free.

We assume that the statement is true for all strong compositions $\beta$ with $\ell(\beta)<\ell$ for an $\ell \geqslant 2$. Let $\alpha=\left(\alpha_{1}, \ldots, \alpha_{\ell}\right)$ be a strong composition with $\ell$ parts, that satisfies Conditions (a), (b) and (c). Since $\operatorname{inv}(\alpha)=0$ implies $\kappa_{\alpha}=\mathfrak{F}_{\alpha}$ by Corollary 15 , we assume that $\operatorname{inv}(\alpha)>0$ and let $i$ be the smallest index such that $\alpha_{i}<\alpha_{i+1}$. We let $T_{1}$ and $T_{2}$ be two quasi-Yamanouchi Kohnert tableaux in $\operatorname{QKT}(\alpha)$ of the same weight. Then we claim that the first row of $T_{1}$ and $T_{2}$ are identical. We deal with two cases $i=1$ and $i>1$ separately to show the claim.

$i=1$ : We will break this part of the proof into three parts. In the first part we will deduce the generic structure of our basic tableau in $\operatorname{QKT}(\alpha)$ when $\alpha_{1}<\alpha_{2}$, resulting in Figure 8. In the second part we will make three crucial observations on producing tableaux in $\operatorname{QKT}(\alpha)$. In the third part we will apply these observations to prove our claim using a proof by contradiction.

Deducing the structure: Note first that since $\alpha_{1}<\alpha_{2}$ and $\alpha$ satisfies Condition (a), $\alpha_{j} \leqslant \alpha_{2}$ for $j>2$. Moreover, if there are $2 \leqslant j<k$ such that $\alpha_{j}<\alpha_{k}$, then $\alpha_{j}$ must be at most $\alpha_{1}$, that is, $\alpha_{j} \leqslant \alpha_{1}$ due to Condition (a).

Let us suppose that $\alpha_{j} \leqslant \alpha_{1}<\alpha_{k}, \alpha_{k+1}$ for $2 \leqslant j<k$. Then because of Condition (a), $\alpha_{l} \leqslant \alpha_{k}$ for all $l>k$ and $\alpha_{k+1}$ is at most $\alpha_{k}$. If $\alpha_{k+1}<\alpha_{k}$, then $1<j<k<k+1$ gives a counterexample of Condition (b) and this implies that $\alpha_{k+1}=\alpha_{k}$. If $\alpha_{1}+1<\alpha_{k+1}=\alpha_{k}$, then $1<j<k<k+1$ is a counterexample of Condition (c) and we can conclude that $\alpha_{k}=\alpha_{k+1}=$ 
$\alpha_{1}+1$. We now suppose that $\alpha_{j}<\alpha_{1}$, then $1<j<k<k+1$ is again a counterexample of Condition (c), hence $\alpha_{j}=\alpha_{1}$.

Consequently the basic tableau looks like Figure 8, where we let $\left\{n_{1}<\right.$ $\left.n_{2}<\cdots<n_{x}\right\}$ be the set $\left\{\alpha_{j} \mid j>2, \alpha_{1}<\alpha_{j}\right\}$ and let $B_{m}=\left\{j \mid \alpha_{j}=n_{m}\right\}$, $m=1,2, \ldots, x$, be the set of rows in the basic quasi-Yamanouchi Kohnert tableau of content $\alpha$ having $n_{m}$ cells.

Note that if $x=1$, then $\alpha_{2}=\alpha_{3}=\cdots=\alpha_{\ell}$ and it is easy to see that by the definition of quasi-Yamanouchi Kohnert tableaux that the claim that the first row of $T_{1}$ and $T_{2}$ are identical is true. Therefore, let us assume that $x>1$.

If $x>1$, then $B_{2} \cup B_{3} \cup \cdots \cup B_{x}=\left\{2,3, \ldots, \max \left(B_{2}\right)\right\}$, that is, there is no row shorter than $\alpha_{1}+1$ among rows $2,3, \ldots, \max \left(B_{2}\right)$ by the definition of $B_{m}$. The yellow box in Figure 8 shows the rows with $\alpha_{1}$ cells. There can be more than one pair of the yellow box consisting of rows of length $\alpha_{1}$ and $B_{1}$ consisting of rows of length $\alpha_{1}+1$. Above that there can be a block that satisfies Conditions (a), (b), (c), of rows of length at most $\alpha_{1}$ at the top of the diagram of $\alpha$. We draw only one pair of such blocks (the yellow box and $B_{1}$ ) in Figure 8 , since the rows above $B_{1}$ do not have any effect on the first row of $T_{1}$ and $T_{2}$ since the entries in these rows cannot be moved to the first row.

Three observations: We now make three crucial observations on creating tableaux in $\operatorname{QKT}(\alpha)$.

i) The first row of a tableau in $\operatorname{QKT}(\alpha)$ is obtained by moving some of the rightmost numbers in the lowest row of each block $B_{m}$ (of the basic tableau) down to the first row so that the contiguous cells that are moved down to the first row from each block is apart by at least one empty cell. This follows by the definition of quasi-Yamanouchi Kohnert tableaux. For example, the gray cells in Figure 8 can be moved down to the first row.

As a consequence we have the next two observations.

ii) If an entry of the lowest row of $B_{1}$ is moved down to the first row of a tableau in $\operatorname{QKT}(\alpha)$, then the only possible moves from rows $r>$ $\max \left(B_{2}\right)$ in the basic quasi-Yamanouchi Kohnert tableau is to move entries either to the first row or within rows between $\max \left(B_{2}\right)+1$ and $\ell$.

iii) If an entry of the lowest row of $B_{m}, m>1$, is moved down to the first row of a tableau in $\operatorname{QKT}(\alpha)$, then it is straightforward to verify 
that the only possible moves from rows $r \geqslant \min \left(B_{m}\right)$ in the basic quasi-Yamanouchi Kohnert tableau is to move entries either down to the first row or no lower than the first row of $B_{m}$.

Concluding identical first rows: Towards a contradiction, suppose that the first row of $T_{1}$ and $T_{2}$ are different and let $m$ be the smallest such that numbers of entries in the first row of $T_{1}$ and $T_{2}$ from $B_{m}$ are different. By our second and third observations we immediately get the following. If $m=1$, then the number of entries in the last rows (above $B_{2}$ ) of $T_{1}$ and $T_{2}$ are different and $T_{1}$ and $T_{2}$ cannot be of a same weight. If $m>1$, then the number of entries in $B_{1} \cup B_{2} \cup \cdots \cup B_{m}$ of $T_{1}$ and $T_{2}$ are different and $T_{1}$ and $T_{2}$ cannot be of a same weight. This contradicts that $T_{1}$ and $T_{2}$ are the same weight. Hence the first row of $T_{1}$ and $T_{2}$ are identical.

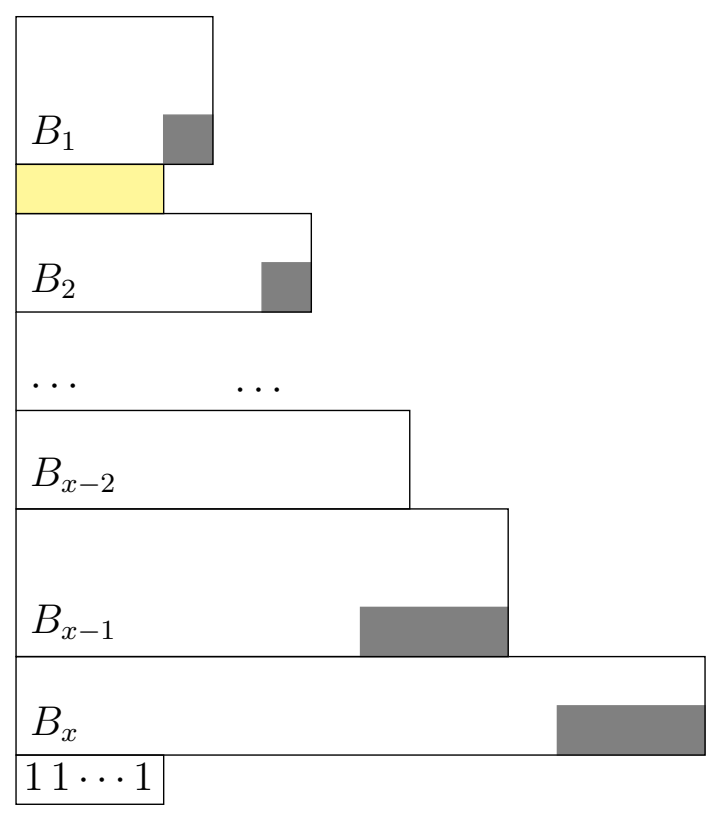

Figure 8: The basic tableau in $\operatorname{QKT}(\alpha)$ when $\alpha_{1}<\alpha_{2}$.

$i>1$ : First note that $\alpha_{j} \leqslant \alpha_{i+1}$ must hold for all $j>i+1$ since $\alpha$ satisfies Condition (a), and $\alpha_{1} \geqslant \alpha_{2} \geqslant \cdots \geqslant \alpha_{i}<\alpha_{i+1}$ by our assumption on $i$. There are two cases to consider:

If $\alpha_{1} \geqslant \alpha_{i+1}$, then the first row of any tableau in $\operatorname{QKT}(\alpha)$ must only contain $\alpha_{1} 1 \mathrm{~s}$.

If not, then $\alpha_{1}<\alpha_{i+1}$ and only $(i+1)$ s can be moved down to the first row of a quasi-Yamanouchi Kohnert tableau. This is because if $j>i+1$ can appear in the first row of a quasi-Yamanouchi Kohnert tableau, then $\alpha_{2} \leqslant$ $\alpha_{1}<\alpha_{j}<\alpha_{i+1}$, by the definition of quasi-Yamanouchi Kohnert tableaux, 
must hold but this violates Condition (b). Therefore, two tableaux of the same weight must have the same first row consisting of 1 s and $(i+1)$ s.

Now, we know that $T_{1}$ and $T_{2}$ have the same first row and we let $T_{1}^{\prime}$ and $T_{2}^{\prime}$ be the tableaux obtained by deleting the first row from $T_{1}, T_{2}$, respectively and subtracting 1 from every remaining entry. Then $T_{1}^{\prime}$ and $T_{2}^{\prime}$ are quasi-Yamanouchi Kohnert tableaux of content $\beta$, where $\beta$ is obtained by subtracting the content $>1$ of the first row of $T_{1}$ (or $T_{2}$ ) from the relevant respective parts of $\alpha$. Then since there is still a cell in every row of the first column of $T_{1}^{\prime}$ and $T_{2}^{\prime}$ it follows that $\beta$ is a strong composition with $\ell(\alpha)-1$ parts. It is straightforward to check that $\beta$ does not contain any of the patterns (a), (b), (c). By the induction hypothesis, $T_{1}^{\prime}$ and $T_{2}^{\prime}$ must be the same tableau and hence we can conclude that $T_{1}$ and $T_{2}$ must be the same tableau too.

When we restrict our attention to strong compositions $\alpha$, we are also able to give an algorithm to produce all the tableaux required to expand a key polynomial as a sum of fundamental slide polynomials, that is, produce all the elements of $\mathrm{QKT}(\alpha)$.

\subsection{Recursive algorithm to produce all elements of QKT $(\alpha)$}

For a strong composition $\alpha=\left(\alpha_{1}, \ldots, \alpha_{\ell}\right)$, let $T_{\alpha}$ be the basic quasi-Yamanouchi Kohnert tableau with content $\alpha$.

- If $\operatorname{inv}(\alpha)=0$, then $\operatorname{QKT}(\alpha)=\left\{T_{\alpha}\right\}$.

- If $\operatorname{inv}(\alpha)=s>0$, then let $i$ be the smallest such that $\alpha_{i}<\alpha_{i+1}$ and let $\hat{\alpha}$ be the strong composition with $\operatorname{inv}(\hat{\alpha})=s-1$, obtained by interchanging the $i$ th and the $(i+1)$ th parts of $\alpha ; \hat{\alpha}=\left(\alpha_{1}, \ldots, \alpha_{i-1}, \alpha_{i+1}, \alpha_{i}, \ldots, \alpha_{\ell}\right)$.

For each $\hat{T} \in \operatorname{QKT}(\hat{\alpha})$, do

- from columns $c=\alpha_{i}+1, \alpha_{i}+2, \ldots$, change all $i$ into $i+1$ and change all $i+1$ into $i$, call the resulting tableau $T_{0}$; let $S(\hat{T}):=\left\{T_{0}\right\}$

- for $k=\alpha_{i}+1, \ldots, \alpha_{i+1}$, do

if the cell in row $(i+1)$ and column $k$ of $T_{0}$ is empty and the cell in row $i$ and column $k$ contains $i+1$, then swap the cells in row $i$ and row $i+1$ from column $\alpha_{i}+1$ to $k$ and note the resulting tableau $T_{k}$; let $S(\hat{T}):=S(\hat{T}) \cup\left\{T_{k}\right\}$.

Theorem 27. For a strong composition $\alpha$, the above algorithm produces all quasi-Yamanouchi Kohnert tableaux of content $\alpha$, that is

$$
\operatorname{QKT}(\alpha)=\bigcup_{\hat{T} \in \operatorname{QKT}(\hat{\alpha})} S(\hat{T}) .
$$

Proof. It is easy to see that given any $T \in \mathrm{QKT}(\alpha)$, we can find a corresponding $\hat{T} \in$ $\mathrm{QKT}(\hat{\alpha})$ by noting the rightmost $i+1$ in row $i+1$ in column $k$ and swapping all cells in row $i$ and row $i+1$ from column $\alpha_{i}+1$ to $k$. Then for all columns $\alpha_{i}+1, \alpha_{i}+2, \ldots$ change all $i$ into $i+1$ and change all $i+1$ into $i$. 
Example 28. Let $\alpha=(2,1,4,3)$. Then $i=2$ and $\hat{\alpha}=(2,4,1,3)$.

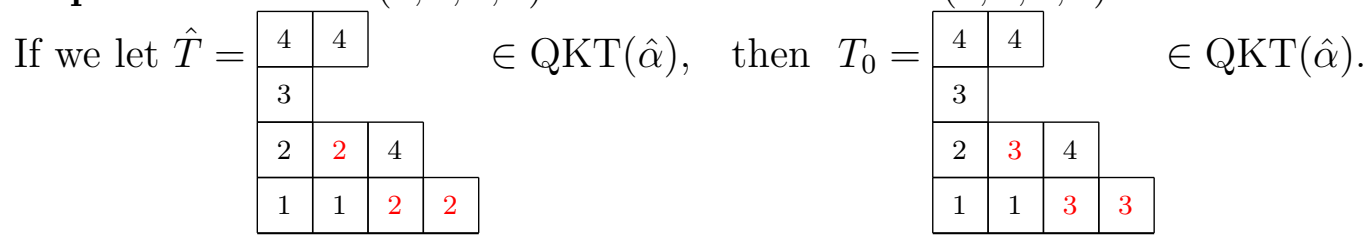

The empty cell in the third row and the second column of $T_{0}$, since the cell below it is filled with $i+1=3$, will make the following quasi-Yamanouchi Kohnert tableau of content $\alpha$.

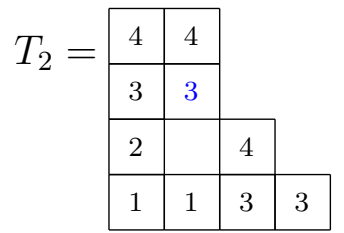

\section{Classifying when $\kappa_{\mathrm{a}}$ is multiplicity free for a a weak composi- tion}

At present classifying in general when a key polynomial is a multiplicity free expansion of fundamental slide polynomials seems substantially more complex than the strong composition case. However, we are able to make progress in some special cases. In particular we focus on the cases related to Theorem 1, namely, hooks and two nonzero parts.

Lemma 29. Let $\alpha$ be a strong composition with $\kappa_{\alpha}$ is not multiplicity free and a be a weak composition with flat $(\mathbf{a})=\alpha$. Then $\kappa_{\mathbf{a}}$ is not multiplicity free.

Proof. If there are two different tableaux of the same weight in $\operatorname{QKT}(\alpha)$, then by adding empty rows and increasing the numbers in the cells by the number of empty rows inserted below we will produce two different tableaux of the same weight in QKT(a).

Lemma 30. If $s_{\mathrm{sort}(\mathbf{a})}$ is multiplicity free, then $\kappa_{\mathbf{a}}$ is multiplicity free.

Proof. Suppose that $\kappa_{\mathbf{a}}$ is not multiplicity free. Then, similar to the proof of Lemma 29, certainly $\kappa_{0^{m} \times \mathbf{a}}$ is not multiplicity free for all $m$ and we can conclude that $s_{\text {sort(a) }}$ is not multiplicity free due to Theorem 6 .

Corollary 31. Let $\mathbf{a}$ be a weak composition with $\operatorname{sort}(\mathbf{a})=\left(n-k, 1^{k}\right)$. Then $\kappa_{\mathbf{a}}$ is multiplicity free.

\subsection{When a has two nonzero parts}

To give an idea of how the complexity increases with the relaxing from strong compositions to weak compositions, note that this case splits into three, depending on whether the number of leading $0 \mathrm{~s}$ is at least two, one or none. 
Theorem 32. For a weak composition a with two nonzero parts and at least two leading $0 s, \kappa_{\mathbf{a}}$ is multiplicity free if and only if

(1) $\operatorname{sort}(\mathbf{a})=\lambda$ for the partitions $\lambda=(3,3),(4,4),(n-2,2)$ for $n \geqslant 4,(n-1,1)$ for $n \geqslant 2$, or

(2) $\operatorname{flat}(\mathbf{a})=(4,3)$.

Proof. Lemma 30 together with Theorem 1 shows that $\kappa_{\mathbf{a}}$ is multiplicity free if $\operatorname{sort}(\mathbf{a})$ is one of $(3,3),(4,4),(n-2,2)$ or $(n-1,1)$. We can check by hand that $\kappa_{\mathbf{a}}$ for $\mathbf{a}=(0,0,4,3)$ and $\mathbf{a}=(0,0,0,4,3)$ is multiplicity free. Moreover, more zeros at the front will keep the key polynomial multiplicity free since by the definition of quasi-Yamanouchi Kohnert tableaux no cell can move to these added rows with 0 cells. Similarly adding zeros between the nonzero parts will keep the key polynomials multiplicity free. Hence, if flat $(\mathbf{a})=(4,3)$ then $\kappa_{\mathbf{a}}$ is multiplicity free.

To prove the other direction, for each of the following Cases (1)-(4) we explicitly find two different tableaux of same weight in $\operatorname{QKT}\left(0,0, \alpha_{1}, \alpha_{2}\right)$. We note that adding zeros at the front does not reduce the multiplicity. Moreover, all tableaux we give can be naturally extended when we add zeros between $\alpha_{1}$ and $\alpha_{2}$, which shows the general cases also.

(1) $\alpha_{1}=\alpha_{2} \geqslant 5$; see Figure 9 .

(2) $\alpha_{1}>\alpha_{2} \geqslant 4$; see Figure 10 .

(3) $\alpha_{1}-1>\alpha_{2}=3$; see Figure 11 .

(4) $\alpha_{2}>\alpha_{1} \geqslant 3$; see Figure 12 .
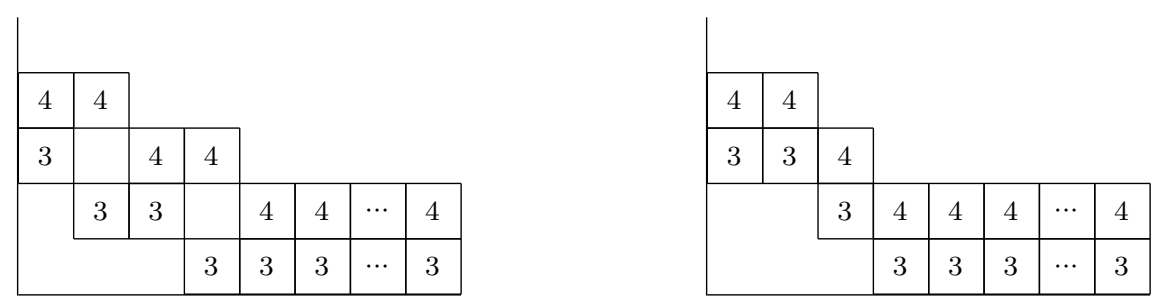

Figure 9: Two tableaux in $\operatorname{QKT}\left(0,0, \alpha_{1}, \alpha_{2}\right)$ of same weight when $\alpha_{1}=\alpha_{2} \geqslant 5$.

In the proof of Theorem 32, two given quasi-Yamanouchi Kohnert tableaux of the same weight for Case (4) given in Figure 12, have empty first rows. This means that there are at least two tableaux in $\operatorname{QKT}\left(0, \alpha_{1}, \alpha_{2}\right)$ when $\alpha_{1}, \alpha_{2}$ satisfy Case (4).

Lemma 33. Let $\mathbf{a}$ be a weak composition with at least one leading 0 and flat $(\mathbf{a})=\left(\alpha_{1}, \alpha_{2}\right)$ for $\alpha_{2}>\alpha_{1} \geqslant 3$. Then $\kappa_{\mathbf{a}}$ is not multiplicity free.

Theorem 34. For a weak composition a with two nonzero parts and exactly one leading $0, \kappa_{\mathbf{a}}$ is multiplicity free if and only if 

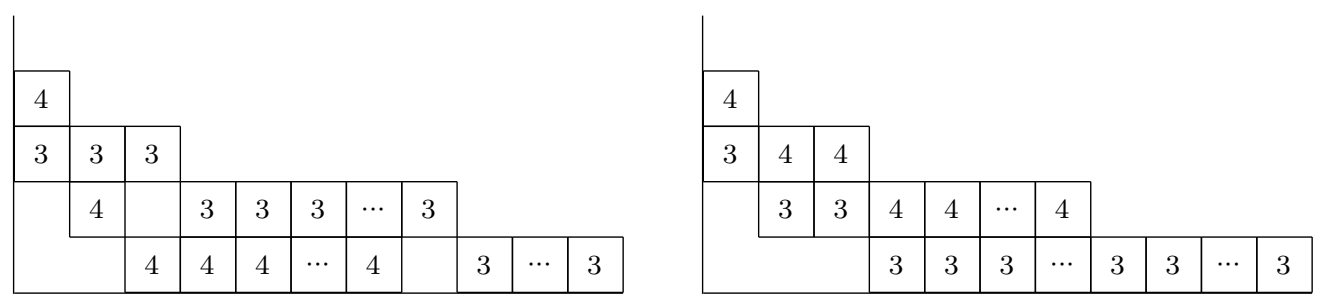

Figure 10: Two tableaux in $\operatorname{QKT}\left(0,0, \alpha_{1}, \alpha_{2}\right)$ of same weight when $\alpha_{1}>\alpha_{2} \geqslant 4$.
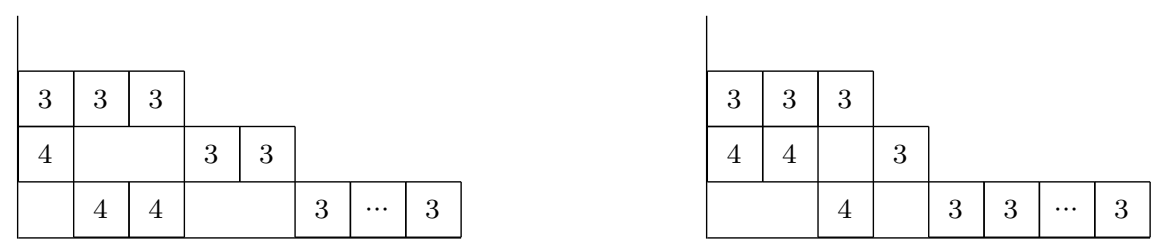

Figure 11: Two tableaux in $\operatorname{QKT}\left(0,0, \alpha_{1}, \alpha_{2}\right)$ of same weight when $\alpha_{1}-1>\alpha_{2}=3$.

(1) $\operatorname{sort}(\mathbf{a})=\lambda$ for the partitions $\lambda=(3,3),(4,4),(n-2,2)$ for $n \geqslant 4,(n-1,1)$ for $n \geqslant 2$, or

(2) flat $(\mathbf{a})=\left(\alpha_{1}, \alpha_{2}\right)$, for $\alpha_{1} \geqslant \alpha_{2}$.

Proof. We know from Lemma 33, that cases other than the ones in the theorem have multiplicities. Hence, we only need to show that in Cases (1) or (2), $\kappa_{\mathbf{a}}$ is multiplicity free.

Lemma 30 together with Theorem 1 shows that $\kappa_{\mathbf{a}}$ is multiplicity free if sort(a) is one of $(3,3),(4,4),(n-2,2)$ or $(n-1,1)$.

Before we prove that weak compositions in (b) are multiplicity free, we note the following: If $T$ is a tableau in $\operatorname{QKT}(0, \alpha_{1}, \underbrace{0, \ldots, 0}_{m}, \alpha_{2})$ for $m>0$, then the row $i, i=$ $3, \ldots, 2+m$, of $T$ is empty, due to the definition of quasi-Yamanouchi Kohnert tableaux. Hence it is enough to consider the weak compositions $\left(0, \alpha_{1}, \alpha_{2}\right)$ for the proof.
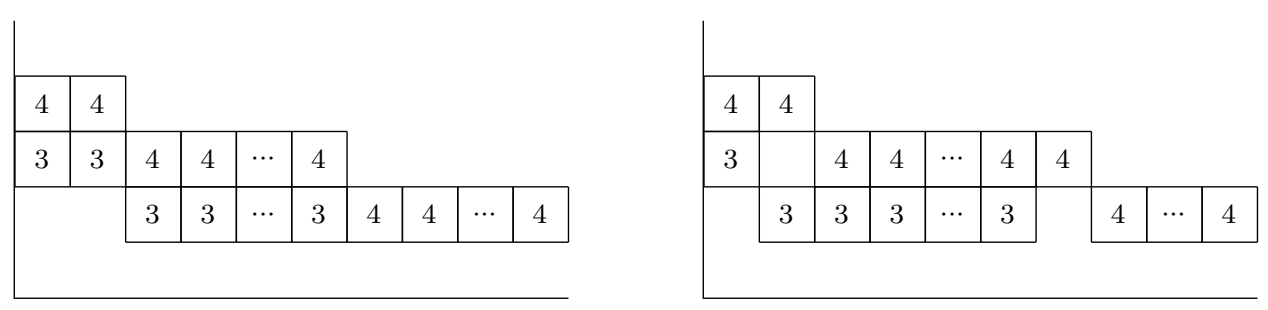

Figure 12: Two tableaux in $\operatorname{QKT}\left(0,0, \alpha_{1}, \alpha_{2}\right)$ of same weight when $\alpha_{2}>\alpha_{1} \geqslant 3$. 
Suppose that $\alpha_{1}=\alpha_{2}=a$. By the definition of (quasi-Yamanouchi) Kohnert tableaux Part (iv) we know that none of the $a 3$ s can appear in row 1 . Thus row 1 will contain $x 2 \mathrm{~s}$, row 3 will contain $y 3 \mathrm{~s}$, and row 2 will contain the remaining $2 \mathrm{~s}$ and $3 \mathrm{~s}$. Since the weight of each of these tableaux is uniquely determined by the number of cells in row 1 and in row 3 , it follows that $\kappa_{(0, a, a)}$ is multiplicity free.

Now suppose that $\alpha_{1}>\alpha_{2} \geqslant 4$, or $\alpha_{1} \geqslant 5$ and $\alpha_{2}=3$. If only $x$, for $1 \leqslant x \leqslant \alpha_{1}$, cells from row 2 move down to row 1 , then we do not obtain a quasi-Yamanouchi Kohnert tableau. If only $y$, for $0 \leqslant y \leqslant \alpha_{2}$, cells from row 3 move down to row 1 , then we obtain a quasi-Yamanouchi Kohnert tableau of weight

$$
\left(y, \alpha_{1}, \alpha_{2}-y\right) \text {. }
$$

If cells from rows 2 and 3 move down to row 1, then, by definition, row 2 has at least $\alpha_{2}+1$ cells but $<\alpha_{1}$ cells, and row 3 has $<\alpha_{2}$ cells. Lastly, if cells from row 2 move down to row 1, and cells from row 3 move down to row 2, then, by definition, row 2 has at most $\alpha_{2}$ cells and row 3 has $<\alpha_{2}$ cells. In every case the weight is unique, and hence $\kappa_{\left(0, \alpha_{1}, \alpha_{2}\right)}$ is multiplicity free.

Finally, $\kappa_{(0,4,3)}$ is multiplicity free since $\kappa_{(0,0,4,3)}$ is multiplicity free as we showed in Theorem 32.

Theorem 35. For a weak composition a with two nonzero parts and no leading $0, \kappa_{\mathbf{a}}$ is multiplicity free.

Proof. Let flat $(\mathbf{a})=\left(\alpha_{1}, \alpha_{2}\right)=\alpha$. If $\alpha_{1} \geqslant \alpha_{2}$, then $\kappa_{\mathbf{a}}$ is multiplicity free by the third part of Theorem 13. If $\alpha_{1}<\alpha_{2}$, then $\kappa_{\alpha}$ is multiplicity free by Lemma 19. Adding zeros between the nonzero parts will keep the key polynomial multiplicity free by definition, and so $\kappa_{\mathbf{a}}$ is multiplicity free.

\section{Acknowledgements}

The authors would like to thank Sami Assaf for posing the question and for many helpful conversations. They would also like to thank the referee for their care and excellent suggestions, and the Korea Institute for Advanced Study where some of this research took place.

\section{References}

[1] S. Assaf and D. Searles, Schubert polynomials, slide polynomials, Stanley symmetric functions and quasi-Yamanouchi pipe dreams, Adv. Math. 306 (2017), 89-122.

[2] _ Kohnert tableaux and a lifting of quasi-Schur functions, J. Combin. Theory Ser. A 156 (2018), 85-118.

[3] C. Bessenrodt, On multiplicity-free products of Schur P-functions, Ann. Comb. 6 (2002), 119-124. 
[4] C. Bessenrodt and S. van Willigenburg, Multiplicity free Schur, skew Schur, and quasisymmetric Schur functions, Ann. Comb. 17 (2013), 275-294.

[5] S. Billey and B. Pawlowski, Permutation patterns, Stanley symmetric functions, and generalized Specht modules, J. Combin. Theory Ser. A 127 (2014), 85-120.

[6] G. Duchamp, D. Krob, B. Leclerc, and J.-Y. Thibon, Fonctions quasi-symétriques, fonctions symétriques non commutatives, et algèbres de Hecke à $q=0, \mathrm{C}$. R. Acad. Sci. Paris 322 (1996), 107-112.

[7] A. Fink, K. Meszaros, and A. St. Dizier, Zero-one Schubert polynomials, Math. Z. (2020).

[8] I. Gessel, Multipartite P-partitions and inner products of skew Schur functions, Combinatorics and algebra (Boulder, Colo., 1983), Contemp. Math., vol. 34, Amer. Math. Soc., Providence, RI, 1984, pp. 289-317.

[9] C. Gutschwager, On multiplicity-free skew characters and the Schubert calculus, Ann. Comb. 14 (2010), 339-353.

[10] R. Hodges and A. Yong, Coxeter combinatorics and spherical Schubert geometry, arXiv:2007.09238.

[11]__ Multiplicity-free key polynomials, arXiv:2007.09229.

[12] B. Ion, Nonsymmetric Macdonald polynomials and Demazure characters, Duke Math. J. 116 (2003), 299-318.

[13] A. Lascoux and M.-P. Schützenberger, Polynômes de Schubert, C. R. Acad. Sci. Paris Sér. I Math. 294 (1982), 447-450.

[14] _ Keys \& standard bases, Invariant theory and tableaux (Minneapolis, MN, 1988), IMA Vol. Math. Appl., vol. 19, Springer, New York, 1990, pp. 125-144.

[15] S. Mason, An explicit construction of type A Demazure atoms, J. Algebraic Combin. 29 (2009), 295-313.

[16] V. Reiner and M. Shimozono, Key polynomials and a flagged Littlewood-Richardson rule, J. Combin. Theory Ser. A 70 (1995), 107-143.

[17] K. Shaw and S. van Willigenburg, Multiplicity free expansions of Schur P-functions, Ann. Comb. 11 (2007), 69-77.

[18] J. Stembridge, Multiplicity-free products of Schur functions, Ann. Comb. 5 (2001), $113-121$.

[19] H. Thomas and A. Yong, Multiplicity-free Schubert calculus, Canad. Math. Bull. 53 (2010), 171-186. 